\title{
Aspectos epidemiológicos das crianças com queimaduras internadas no Pronto Socorro para
} Queimaduras de Goiânia - Goiás

\author{
Epidemiologic aspects of children with burns hospitalized in the First Aid Clinic for \\ Burns of Goiânia - Goiás
}

\author{
Aspectos epidemiológicos de los niños con quemaduras hospitalizados en Primeros Auxilios para \\ Quemaduras de Goiânia - Goiás
}

Fabiana Pavan VianaI ${ }^{I}$ Selma Mendes ResendeII, Márcia Claudina Tolêdo ${ }^{\mathrm{III}}$, Roberta Cordeiro Silva ${ }^{\mathrm{IV}}$

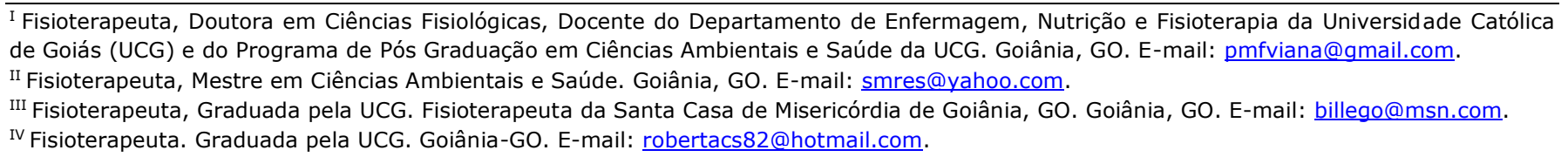

\section{RESUMO}

As crianças são as vítimas mais frequentes das queimaduras e estas a segunda causa de morte acidental na infância. O objetivo deste trabalho foi investigar as características epidemiológicas de crianças de 0 a 14 anos, vítimas de queimaduras, internadas no Pronto Socorro para Queimaduras de Goiânia - Goiás. Para isto, dados obtidos no Banco de Dados do hospital e prontuários das crianças internadas no período de $1^{\circ}$ de junho de 2005 a $1^{\circ}$ de junho de 2007 foram analisados. As crianças foram divididas em dois grupos: Grupo I (0-4 anos) e Grupo II (5-14 anos). Em ambos os grupos o sexo masculino foi predominante e a maioria dos atendimentos ocorreu via convênio do hospital com o Sistema Único de Saúde. O principal local dos acidentes foi na residência. O agente causal mais comum no Grupo I foram os líquidos superaquecidos e no Grupo II, as chamas $(p<0,001)$. Quarenta e dois por cento das crianças tiveram de 10 a $19 \%$ da superfície corporal queimada. Sugere-se a inclusão de programas educativos na comunidade, nas Unidades Básicas de Saúde, nas escolas de educação infantil e creches, que auxiliem na conscientização dos cuidadores quanto às formas de prevenção das queimaduras.

Descritores: Epidemiologia; Queimaduras; Criança.

\section{ABSTRACT}

Children are the most frequent victims of burns, and these are the second cause of accidental infant mortality. The objective of this work was to investigate the epidemiologic characteristics of children between 0 and 14 years old, victims of burns, hospitalized in the First Aid Clinic for Burns of Goiânia - Goiás. For that, data from the database of the hospital and registries of the hospitalized children in the period of June $1^{\text {st }}, 2005$ to June $1^{\text {st }}, 2007$ were analyzed. The children were divided in two groups: Group I (0-4 years old) and Group II (5-14 years old). In both groups the male sex was predominant and the majority of attendances happened through accord between the hospital and the Single Healthcare System. The main accident place was at home. The most common causal agent in Group I were the overheated liquids and in Group II, the flames ( $p<0.001$ ). Forty-two percent of the children had from 10 to $19 \%$ of the body surface burnt. It is suggested to include educative programs in the community, in the Basic Healthcare Units, in infant education schools and nursery schools, to help awareness of carers about how to prevent burns.

Descriptors: Epidemiology; Burns; Child.

\section{RESUMEN}

Los niños son las víctimas más frecuentes de las quemaduras y estas son la segunda causa de muerte accidental en la infancia. El objetivo de este trabajo fue identificar las características epidemiológicas de niños de 0 a 14 años, víctimas de quemaduras, internadas en el Primero Auxilio para Quemaduras de Goiânia - Goiás. Para esto, se analizaron los datos en la base de datos de los registros hospitalarios y médicos de los niños hospitalizados entre el 1 de junio de 2005 y 1 de junio de 2007. Los niños fueron divididos en dos grupos: Grupo I (0-4 años) y Grupo II (4-14 años). En ambos grupos el sexo masculino fue predominante. La mayoría de las atenciones fueron realizadas por convenio entre el hospital y el Sistema Único de Salud. El principal lugar de los accidentes fue en la residencia. La causa más común en el Grupo I fueron los líquidos extremadamente calientes, y en el Grupo II, las llamas $(p<0,001)$. El cuarenta y dos por ciento de los niños sufrieron quemaduras entre el $10 \%$ y el $19 \%$ de la superficie corporal. Se sugiere incluir programas educativos en la comunidad, Unidades Básicas de Salud, escuelas de educación infantil y guarderías, para auxiliar la concientización de los custodios como medios para la prevención de quemaduras.

Descriptores: Epidemiología; Niño; Quemaduras. 


\section{INTRODUÇÃO}

Os acidentes representam um dos principais problemas de saúde pública, destacando-se entre esses a queimadura(1). A queimadura é uma lesão causada por agentes térmicos, químicos, elétricos ou radioativos que agem no tecido de revestimento do corpo humano e pode destruir parcialmente ou totalmente a pele, seus anexos e até atingir camadas mais profundas, como os tecidos subcutâneos, músculos, tendões e ossos ${ }^{(1)}$.

Estudos epidemiológicos em diversos países indicam que as queimaduras representam uma das principais causas não intencionais de acidentes em crianças e adolescentes ${ }^{(2-4)}$. Essas representam a segunda causa de morte acidental na infância(5). Nos países em desenvolvimento as queimaduras representam $96 \%$ das causas de morte por lesão unidirecional, totalizando 271.000 mortes $^{(6)}$. No Brasil, em 2001, ocorreu um milhão de acidentes por queimaduras e aproximadamente 2.500 destes indivíduos foram a óbito(6).

Nos Estados Unidos da América os custos diários com o tratamento hospitalar para as vítimas de queimaduras variam entre 3.000 e 5.000 dólares $^{(7)}$. Além disso, outros fatores como a perda de dias de trabalho, as incapacidades, a reabilitação física e emocional oneram o cuidado com estas vítimas ${ }^{(6)}$.

As crianças apresentam maior predisposição para acidentes. Nesse período de desenvolvimento, a criança é curiosa, inquieta, inexperiente, exploradora, ativa e, na maioria das vezes é incapaz de identificar e avaliar o perigo(8). Estes fatores associados à negligência dos familiares facilitam os acidentes ${ }^{(2)}$.

Pesquisas realizadas no Brasil e em diversos países apontam que os principais agentes etiológicos dos acidentes por queimaduras em crianças são os escaldamentos por água quente, líquidos quentes e os produtos inflamáveis. E quanto ao sexo, os meninos são mais propensos a este tipo de injúria ${ }^{(9-}$ 10).

Os acidentes por queimaduras são considerados dos mais graves traumas, pois além do risco de morte, as complicações como septicemia, falência renal e cardio- respiratória, poderão induzir importantes modificações metabólicas, sequelas físicas e psicológicas ${ }^{(11)}$.

Atualmente a maioria dos acidentes e das mortes em crianças causadas por queimaduras podem ser evitadas. Deste modo, ações preventivas são necessárias. A atuação conjunta envolvendo setores do governo e da população civil poderá reduzir de modo significativo os acidentes e os elevados custos de hospitalização e recuperação. Contudo para prevenir é necessário conhecer a magnitude do problema, identificar as populações mais atingidas e as circunstâncias nas quais as queimaduras ocorreram ${ }^{(9)}$. Entretanto, poucos estudos discutem os aspectos epidemiológicos de crianças vítimas de queimaduras internadas no Brasil( $(4,12)$ e especificamente no Estado de Goiás.

De acordo com as premissas anteriores os objetivos deste trabalho foram investigar as características epidemiológicas de crianças de 0 a 14 anos, vítimas de queimaduras, internadas no Hospital de Tratamento de Queimaduras de Goiás e verificar a diferença das características epidemiológicas dos indivíduos estudados em diferentes faixas etárias.

\section{MÉTODOS}

Foi realizado um estudo epidemiológico, retrospectivo. Foram incluídas todas as crianças até 14 anos internadas no Pronto Socorro para Queimaduras de Goiânia - GO por um período de dois anos (10 de junho de 2005 a $1^{\circ}$ de junho de 2007). Os dados foram coletados nos registros do Banco de Dados do hospital, e para complementar a coleta os prontuários arquivados foram analisados.

As variáveis estudadas foram: o sexo, a idade, a procedência, o local do acidente, os agentes causais, a área queimada, os óbitos e se o atendimento foi realizado pelo Sistema Único de Saúde ou via plano de saúde e particular. Foi estabelecida a subdivisão das crianças segundo a classificação etária do IBGE (Instituto Brasileiro de Geografia e Estatística), sendo - Grupo I de zero a quatro anos e o Grupo II, de cinco a 14 anos com a finalidade de observar diferenças epidemiológicas, uma vez que estes grupos estão em diferentes estágios de desenvolvimento físico-funcional, e esse pode interferir nos diversos fatores de riscos que os predispõem as queimaduras. Este trabalho foi aprovado pelo Comitê de Ética e Pesquisa da Universidade Católica de Goiás sob protocolo 0040.0.168.000-07.

$\mathrm{Na}$ análise utilizou-se para a realização dos cálculos estatísticos o programa Statistical Package for the Social Sciences (SPSS), versão 14.0. Na análise estatística descritiva foi verificada a distribuição das diferentes faixas (Grupo I e Grupo II) pela frequência das variáveis acima citadas. Para comparação das variáveis nominais, utilizou-se o Teste Qui-Quadrado e o Teste Exato de Fischer quando necessário, nas correlações definidas. O nível de significância adotado foi $\square=0,05$.

\section{RESULTADOS E DISCUSSÃO}

Após análise dos dados pode-se constatar que no período estudado foram internadas 410 crianças, sendo $236(57,6 \%)$ pertencentes a faixa etária de zero a quatro anos, $263(64,1 \%)$ do sexo masculino e $267(65,1 \%)$ procedentes do interior de Goiás (Tabela 1). 
Tabela 1: Características das crianças com queimaduras internadas no Pronto Socorro para Queimaduras de Goiânia - GO, 2005-2007.

\begin{tabular}{lcc}
\hline \multicolumn{1}{c}{ Característica } & $\mathbf{n}$ & $\mathbf{\%}$ \\
\hline Faixa etária & & \\
$\quad$ Grupo I (0 - 4 anos) & 236 & 57,6 \\
$\quad$ Grupo II (5 - 14 anos) & 174 & 42,4 \\
Sexo & & \\
$\quad$ Masculino & 263 & 64,1 \\
$\quad$ Feminino & 147 & 35,9 \\
Procedência & & \\
$\quad$ Região metropolitana (Goiânia) & 126 & 30,8 \\
$\quad$ Interior do Estado de Goiás & 267 & 65,1 \\
$\quad$ Outros Estados & 17 & 4,1 \\
\hline
\end{tabular}

$\mathrm{p}<0,001$ ao se comparar predominância do sexo masculino

$\mathrm{Na}$ pesquisa realizada pode-se averiguar que as queimaduras foram mais frequentes nas crianças menores de cinco anos. Estes achados corroboram os resultados obtidos por diversos autores ${ }^{(3-4,11,13)}$. É provável que este resultado esteja relacionado à fase de desenvolvimento em que estas crianças se encontram, uma vez que neste período são ávidas por novas descobertas, exploram o meio ambiente, entretanto não têm consciência do perigo a que estão expostas. Este fato predispõe-nas a sofrerem acidentes. Além disso, muitos destes ocorrem devido à negligência dos adultos no cuidado com as crianças $^{(8)}$.

As crianças do sexo masculino foram significativamente mais suscetíveis aos acidentes por queimaduras que aquelas do sexo feminino nos dois grupos estudados $(p<0,001)$. Sexo Masculino: Grupo I: $63,1 \%$ (149) e Grupo II: 65,5\% (114); sexo feminino: Grupo I: $36,9 \%$ (87) e Grupo II: 34,5\% (60). Não foram observadas diferenças significativas entre os grupos analisados quanto ao sexo $(p=0,619)$. Este resultado é semelhante aos estudos obtidos por diversos autores ao caracterizarem o perfil epidemiológico de crianças vítimas de queimaduras ${ }^{(9-10,14)}$. Possivelmente, isto ocorre porque os meninos apresentam um comportamento mais arrojado, uma vez que exploram de modo abrangente o meio ambiente no qual estão inseridos e consequentemente se tornam mais predispostos aos acidentes.

Ao analisar a procedência das crianças acidentadas, observou-se que a maioria é do interior de Goiás. Este resultado é justificado pela ausência de instituições especializadas e descentralizadas no interior do Estado para o tratamento de queimados.

Quanto ao local da queimadura, a residência foi o mais referenciado com $362(88,3 \%$ ) casos (Tabela $2)$, dados estes encontrados por outros autores ${ }^{(15-16)}$. Para estes pesquisadores, a maior parte dos acidentes por queimaduras provém dos ambientes domiciliares, e acontece especialmente na cozinha, onde estão localizados os principais agentes causadores. No presente trabalho não foi possível obter o local do domicílio em que as queimaduras ocorreram, uma vez que este dado não constava nos prontuários.

Tabela 2: Local do acidente ocorrido com as crianças com queimaduras internadas no Pronto Socorro para Queimaduras de Goiânia - GO, 2005-2007.

\begin{tabular}{ccc}
\hline Local do Acidente & $\mathbf{n}$ & \% \\
\hline Residência & 362 & 88,3 \\
Trânsito & 24 & 5,8 \\
Lazer & 9 & 2,2 \\
Outros & 15 & 3,7 \\
\hline
\end{tabular}

Os agentes causais que mais provocaram hospitalização nas crianças do Grupo I foram os líquidos superaquecidos e nas crianças do Grupo II foram as chamas (Tabela 3). Observaram-se diferenças significativas dos agentes causais entre os grupos estudados $(p<0,001)$. 
Tabela 3: Agentes causadores das lesões das crianças com queimaduras internadas no Pronto Socorro para Queimaduras de Goiânia - GO, 2005-2007.

\begin{tabular}{ccccc}
\hline Agentes & \multicolumn{2}{c}{ Grupo I (0 a 4 anos) } & \multicolumn{2}{c}{ Grupo II (5 a 14 anos) } \\
& $\mathbf{n}$ & $\mathbf{\%}$ & $\mathbf{n}$ & $\mathbf{\%}$ \\
\hline Líquidos Quentes & 159 & 67,4 & 53 & 30,5 \\
Chamas & 27 & 11,4 & 72 & 41,4 \\
Superfícies Aquecidas & 21 & 8,9 & 12 & 6,9 \\
Atrito & 8 & 3,4 & 20 & 11,5 \\
Eletricidade & 14 & 5,9 & 8 & 4,6 \\
Químicos & 5 & 2,1 & 5 & 2,9 \\
Aderentes & 2 & 0,8 & 4 & 2,3 \\
\hline Total & $\mathbf{2 3 6}$ & $\mathbf{1 0 0}$ & $\mathbf{1 7 4}$ & $\mathbf{1 0 0}$ \\
\hline
\end{tabular}

$\mathrm{p}<0,001$ ao se comparar os agentes causais entre os grupos I e II

Segundo os resultados obtidos observou-se associação estatisticamente significativa entre a idade e os agentes etiológicos. Os líquidos aquecidos foram os principais causadores de queimaduras em crianças de 0 a 4 anos. Estes resultados estão de acordo com as pesquisas de outros autores $(3,7,11,13,17)$. Uma provável explicação para este acontecimento é que as crianças nesta idade, de acordo com o desenvolvimento psicomotor, estão iniciando o processo de engatinhar e as primeiras passadas, e estas associadas à curiosidade inerente em explorar o espaço em sua volta, acabam aproximando-se de locais como a cozinha onde há bebidas, alimentos, óleo e outros líquidos quentes, que ao serem entornados sobre a criança atingem principalmente tronco, ombro, braço e antebraço; quadril, coxa e perna e, também, cabeça e pescoço( ${ }^{(6)}$.

Já as queimaduras causadas por chamas foram mais frequentes na faixa etária compreendida de 5 a
14 anos, semelhante aos resultados apresentados por outros autores $(7,18)$. Nesta idade são comuns acidentes envolvendo o uso do álcool etílico, sendo este produto utilizado no Brasil em ambiente doméstico para limpeza e também como substância inflamável. Muitas vezes o álcool permanece ao alcance das crianças, que devido à falta de vigilância e fiscalização de um adulto acabam sendo acometidas. As queimaduras provocadas por álcool são mais profundas que as provocadas por água fervente, pois o tempo de exposição da pele ao calor em queimaduras causadas por líquido inflamável é maior.

Quanto à área queimada, 42,2\% tiveram de $10 \mathrm{a}$ $19 \%$ da superfície corporal queimada e $1,7 \%$ queimaram mais que $50 \%$ da área corporal (Tabela 4). Não foram observadas diferenças significativas da área queimada entre os Grupos I e II $(p=0,304)$.

Tabela 4: Superfície corporal queimada das crianças internadas no Pronto Socorro para Queimaduras de Goiânia - GO, 2005-2007.

\begin{tabular}{|c|c|c|c|c|}
\hline \multirow{2}{*}{ Área Queimada } & \multicolumn{2}{|c|}{ Grupos I ( 0 a 4 anos) } & \multicolumn{2}{|c|}{ Grupo II (5 a 14 anos) } \\
\hline & $\mathbf{n}$ & $\%$ & $\mathbf{n}$ & $\%$ \\
\hline 0 a $9 \%$ & 71 & 30,1 & 60 & 34,5 \\
\hline 10 a $19 \%$ & 102 & 43,2 & 71 & 40,8 \\
\hline 20 a $29 \%$ & 46 & 19,5 & 31 & 17,8 \\
\hline 30 a $39 \%$ & 11 & 4,7 & 3 & 1,7 \\
\hline 40 a $49 \%$ & 4 & 1,7 & 4 & 2,3 \\
\hline$\geq 50 \%$ & 2 & 0,8 & 5 & 2,9 \\
\hline Total & 236 & 100 & 174 & 100 \\
\hline
\end{tabular}

$p=0,304$ ao se comparar a superfície das áreas queimadas entre os grupos I e II

A média de 10 a $19 \%$ de superfície corporal queimada para a maioria dos investigados é semelhante aos obtidos em outros estudos(19-20). Cabe lembrar que as crianças têm uma maior superfície corporal em relação ao peso ao se comparar com um adulto. Logo, uma criança que apresenta $20 \%$ da superfície corporal queimada é considerada um grande queimado(6).

Das crianças estudadas, $365(89,1 \%)$ foram atendidas por meio do Sistema Único de Saúde (SUS). Os gastos públicos no tratamento de pacientes queimados poderiam ser reduzidos por meio de campanhas preventivas que garantissem uma atenção primária, assegurada a todos os setores da sociedade, em especial aos pais e as crianças.

No período analisado, sete crianças evoluíram para óbito, sendo quatro do Grupo I e três do Grupo II. Mas, nas últimas décadas tem sido observada maior taxa de sobrevida e possivelmente este resultado é decorrente da melhor compreensão da fisiopatologia da queimadura, assim como do tratamento precoce das lesões. 
A partir das premissas anteriores, sugere-se a implantação, inserção de trabalhos educativos e preventivos com atividades específicas para a temática em questão. Os recursos podem ser cartazes, folhetos explicativos, palestras, meios de comunicação (televisiva ou falada), que orientem a população sobre os cuidados a serem tomados, principalmente com as crianças abaixo de quatro anos no que se refere aos líquidos aquecidos, e com as maiores, quanto aos inflamáveis.

\section{CONCLUSÃO}

Após a análise dos dados pode-se constatar que os acidentes foram predominantes no sexo masculino. O principal local dos acidentes foi na residência. O agente causal mais comum no Grupo I foram os líquidos superaquecidos e no Grupo II, as chamas $(p<0,001)$. Quarenta e dois por cento das crianças tiveram de 10 a $19 \%$ da superfície corporal queimada. Desta forma, tornam-se necessários programas educativos na comunidade, nas Unidades Básicas de Saúde, por meio dos agentes comunitários de saúde, nas escolas de educação infantil ou creches, na busca de conscientizar pais e educadores da necessidade de atenção, de modo a evitar o contato dos infantes com substâncias inflamáveis ou outros agentes que causem as queimaduras.

A maioria dos atendimentos ocorreu via convênio do hospital com o Sistema Único de Saúde. Sendo assim, uma política pública para redução de acidentes domésticos com queimaduras também contribuiria para redução de gastos públicos na área da saúde.

\section{REFERÊNCIAS}

1. Kara IG, Gök S, Horsanli O, Zenair M. A population-based questionnaire study on the prevalence and epidemiology of burn patients in Denizli, Turkey. J. Burn. Care Res. 2008;29(3):44650.

2. Chester DL, Jose RM, Aldlyami E, King $H$, Moiemen NS. Non-accidental burns in children - are we neglecting neglect? Burns. 2006;32(2):222-8.

3. Frans FA, Keli SO, Maduro AE. The epidemiology of burns in a medical center in the Caribbean. Burns. 2008;34(8): 1142-18.

4. Machado THS, Lobo JA, Pimentel PCM, Serra MCVF. Estudo epidemiológico das crianças queimadas de 0-15 anos atendidas no Hospital Geral do Andaraí, durante o período de 1997 a 2007. Rev. Bras. Queim. 2009;8(1):3-8.

5. Rossi LA, Ferreira E, Costa EC, Brgamasco EC, Camargo C. Prevenção de queimaduras: percepção de pacientes e seus familiares. Rev Latino-am Enfermagem. 2003;11(1):36-42.

6. Júnior EML, Serra MCVF. Tratado de Queimaduras. São Paulo: Atheneu; 2004.
7. Papp A, Rytkönen $T$, Koljonen V, Vuola J. Paediatric ICU burns in Finland 1994-2004. Burns. 2008;34(3):339-44.

8. Silva HTS, Almeida JS, Souza SIF, Costa IMP. Queimaduras: um estudo de caso na unidade de tratamento de queimados do hospital público do oeste, em Barreiras - BA. Revista Digital de Pesquisa CONQUER da Faculdade São Francisco de Barreiras. 2008 [cited 2009 dez 30];3. Available from: http://www.fasb.edu.br/revista/index.php/conquer/ar ticle/viewFile/84/61.

9. Maghsoudi H, Pourzand UM, Azarmir G. Ettiology and outcome of burns in Tabriz, Iran: a analysis of 2963 cases. Scand. J. Sug. 2005;94(1):77-81.

10. Rocha HJS, Lira SVG, Abreu RNDC, Xavier EP, Vieira LJES. Perfil dos acidentes por líquidos aquecidos em crianças atendidas em Centro de Referênciade Fortaleza. RBPS. 2007;20(2):86-91.

11. Sharma PN, Bang RL, Al-Fadhli AN, Sharma P, Bang S, Ghoneim IE. Paediatric burns in Kuwait: incidence, causes and mortality. Burns. 2006;32(1):104-11.

12. Bernz LM, Mignoni ISP, Pereima MJL, Souza JA, Araújo EJ, Feijó R. Análise das causas de óbitos de crianças queimadas no Hospital Infantil de Gusmão, no período de 1991 a 2008. Rev Bras Queimaduras. 2009;8(1):9-13.

13. Serour F, Gorenstein A, Boaz M. Characteristics of thermal burns in children admitted to an Israeli pediatric surgical ward. Isr Med Assoc J. 2008;10(4):282-6.

14. Shields BJ, Comstock RD, Fernandez SA, Xiang $H$, Smith GA. Healthcare resource utilization and epidemiology of pediatric burn-associated hospitalizations, United States, 2000. J. Burn. Care Res. 2007;28(6):811-26

15. Pardo GD, García IM, Marrero FRM, Cía TG. Psychological impact of burns on children treated in a severe burns unit. Burns. 2008;34(7):986-93.

16. Haik J, Liran A, Tessone A, Givon A, Orenstein A, Peleg K. Burns in Israel: demographic, etiologic and clinical trends, 1997-2003. Isr Med Assoc J. 2007;9: 659-62.

17. Arshi S, Sadeghi-Bazargani H, Mohammadi R, Ekman R, Hudson D, Djafarzadeh $\mathrm{H}$ et al. Prevention oriented epidemiologic study of accidental burns in rural areas of Ardabil, Iran. Burns. 2006;32(3):36671.

18. Senel E, Yasti AC, Reis E, Doganay M, Karacan $C D$, Kama NA. Effects on mortality of changing trends in the management of burned children in Turkey: eight years' experience. Burns. 2009;35(3):372-77.

19. Silva MCC, Pacheco JS, Furtado FVS, Matos Filho JC, Damasceno AKC. Epidemiologia das infecções em queimaduras no nordeste do Brasil. Rev. Eletr. Enf. [Internet]. 2009 [cited 2009 dez 30];11(2):390-4. 
Available

from:

http://www.fen.ufg.br/revista/v11/n2/pdf/v11n2a21.pdf 20. Barbosa MINH, Gomes DR, Serra MCVF, Guimarães Junior LM, Varges Filho RC, Muniz RT et al. Queimaduras em crianças e adolescentes: análise de 1302 casos. Rev Bras Queimaduras. 2002;2(1):25-30.

Artigo recebido em 02.10.08.

Aprovado para publicação em 22.09.09.

Artigo publicado em 31.12.09. 